\title{
Syntactic Structures in the Albanian Translation of Dreiser
}

\author{
Dr. Gentjana Taraj \\ Kristal University, Tirana \\ Dr. Helena Grillo \\ Aleksander Moisiu University, Durrës
}

\section{Doi:10.5901/mjss.2013.v4n11p548}

\begin{abstract}
Social sciences are increasingly oriented towards interdisciplinary studies. Intercultural communication, an interdisciplinary field of research, examines the way people communicate and understand each other across boundaries and various types of discurse systems, and the extent to which they affect the use of language (Richards \& Schmidt, 1983:265). In the context of intercultural communication, the current paper explores the effect of linguistic structures on the communication, mainly the written language. Literary translation is probably the most debated form of translation, which constitutes $60 \%$ of the literary publication in Albania. Literary translators tend to uncover what lies under the surface which often requires going beyond the boundaries of the language. The focus of this paper is on the grammatical structure and the possibility of the Albanian language to express Dreiser's works fluently and accurately. The paper compares and contrasts the syntactic construction of complex sentences in both languages. The paper concludes with the idea that literary translators have to adopt sentences and their clauses from the source language to make reference to the original context. Furthermore, an attempt has been made to introduce some suggestions for a better translation.
\end{abstract}

Keywords: intercultural communication, syntactic structures, translation, equivalence, literary works.

\section{Introduzione}

La parola "traduzione" viene dal latino translation che significa condurre, portare o trasferire una cosa da una parte ad un'altra. In altre parole tradurre vuol dire di esprimere lo stesso significato con una forma equivalente ad un altra. La prosa rappresenta la scrittura usuale senza una struttura metrica o come si suol dire "le parole nel loro miglior ordine"(Webster, 1913). In conseguenza la traduzione della prosa si puo considerare un genere di attivita creativa che riscrive l'opera scritta in una lingua in un altra lingua. Secondo Peter Newmark la traduzione è "dare di un testo in lingua straniera il significato corrispondente nella nostra lingua come l'autore del testo originale lo aveva inteso"(Newmark, 1988:5). Da questo si evince che è compito del traduttore di considerare il testo nella sua interezza in quanto la traduzione non si riduce nel semplice tentativo di riportare nel testo tradotto il significato ma di riportare altretanto elementi del sottotesto. La traduzione ha come punto di partenza il testo originale, ma il traduttore per riscriverlo deve saper usare i mezzi espressivi e linguistici che ofre la lingua di arrivo. II traduttore letterario deve essere in grado non solo di tradurre il significato del testo ma di trasmettere i sentimenti, le sfumature culturali, lo spirito del testo originale.

Nel processo di assaggio e comprensione del testo originale, Nida (1964) distingue tra l'equivalenza formale e l'equivalenza dinamica. Se l'equivalenza formale concentra l'attenzione sia sulla forma che sul significato del messaggio, l'equivalenza dinamica si basa sul principio del effetto equivalente. Secondo Nida l'equivalenza dinamica non comporta solo la comunicazione del messaggio ma "è la riproduzione nella lingua di arrivo dell'equivalente naturale più prossimo al messaggio comunicato nella lingua di partenza" (Nida \& Taber, 1982:200). Su questa definizione discussa a lungo sono presenti tre parole chiave: equivalenza, la quale si riferisce al messaggio nella lingua di partenza, b) naturale, la quale si riferisce alla lingua, alla cultura, al contesto e al destinatario del messaggio nella lingua di arrivo e c) più prossimo la quale mette in relazione entrambe le lingue.

\section{Autore/Libro/Lettore/=Traduttore/Libro/Lettore}

Fig.1 II rapporto tra l'autore e il traduttore rispetto al testo e al lettore 
Nel caso in cui la lingua di partenza e la lingua d' arrivo non appartengono allo stesso gruppo culturale(il caso della lingua albanese e quella inglese) il traduttore deve tenere conto dei translation shifts, che J.C.Catford (1965:73) descrive come "deviazione dalla corrispondenza formale nel procedere da una lingua di partenza ad una lingua d'arrivo". Catford distingue due tipi di translation shifts: (1) level shifts (quando ciò che è espresso da mezzi grammaticali in una lingua viene trasposto con mezzi lessicali in un'altra) e (2) category shifts suddivisi in quattro tipi: (a) structure-shift (i cambiamenti più comuni che investono generalmente le strutture grammaticali), (b) unit shifts (una espressione puo essere tradotta con una parola), (c) class shifts (un aggettivo puo essere tradotto con un nome) e (d) intra-system shifts (il nome al singolare nella lingua di partenza puo essere tradotto con un nome al plural nella lingua d-arrivo). In questo contesto, gli aspetti grammaticali, diversi per ambedue le lingue, sono un oggetto interesante su cui indagare. In coseguenza le domande alle quali cercheremo di rispondere sono: quali sono i tipi di equivalenza e e quali sono i shifts usati nella traduzione, dall'inglese in albanese, del periodo semplice e del periodo complesso nell' opera di Dreiser?

\section{La frase complessa}

Nel processo di selezione delle strutture sintattiche in un opera letteraria hanno un ruolo determinante una serie di fattori, come: "il carattere dell'ogetto descritto, il tono emozionale che l'autore intende dare al suo racconto, la tradizione dalla quale lui parte, le caratteristiche del tipo di prosa che scrive ecc"(Bulo, 1984:160). Tra di essi rivestono particolare importanza quelli collegati con il contenuto. La concezione ideologica dell'autore, il suo modo di pensare e di percezione della realtà cosi come la problematica esposta sono i fattori che definiscono l'orientamento e i principi dell'autore nella costruzione stilistica del discorso artistico. In questo modo nelle opere ad esempio in cui "domina il tono epico, è carateristico lo stile analitico dell'esposizione accompagnato da configurazioni complesse e strutture elaborate"(Bulo, 1984:161). Secondo Bulo, all'intezione dell autore di rappresentare la vita attraverso le relazioni causali degli eventi, la penetrazione in ogni suo nodo e l'elaborazione degli eventi complessi dello sviluppo sociale e storico corrisponde l'uso di "strutture sintattiche caratterizzate da periodi composti e complessi costruite con congiunzioni coordinati e subordinati e con una posizione generalmente libera delle parole nelle frasi"(Quirk, Greenbaum, Leech, \& Svartvik, 1987).

Le opere di Dreiser si distinguono per l'uso delle frasi complesse che dimostrano la maturità nel suo pensiero. In inglese le frasi complesse si suddividono in diverse categorie a secondo il tipo della frase subordinata. La frase complessa è l'unione di almeno una frase semplicie principale con una subordinata. Le frasi subordinate possono essere finite e non-finite (Hadaj, 1984). Le frasi subordinate finite si suddividono in: frasi subordinate avverbiali, frasi subordinate sostantive, frasi subordinate Wh e frasi subordinate relative. Le frasi subordinate non-finite sono quelle ottenute con l'uso dei modi indefiniti come infinitive o participle. In albanese le frasi si suddividono in due categorie principali: (1) frasi semplici e (2) frasi complesse. La frase complessa coordinata e' formato da due frasi indipendenti coordinate tra loro. La frase subordinata è formato da due o più frasi di cui una è la principale e le altre sono subordinate ad essa. Secondo il tipo della frase subordinata, le frasi complesse possono essere: temporale, locutiva, causale, condizionale ecc.

\section{L' equivalenza dinamica e formale}

Ogni lingua segmenta in maniera diversa la realtà utilizzando strutture grammaticali, regole grammaticali e diverse variazioni sintattiche, motivo per cui la traduzione rappresenta una sfida. Per esprimere l'insieme dell'informazione estetica e semantica del testo originale, il traduttore cerca di utilizzare con maestria non solo i lessico ma anche la sintassi della lingua d'arrivo (Belloc,1931), perchè la scarsa familiarità con esse puo dare origine a costruzioni linguistiche in contradizione con la natura stessa della lingua d'arrivo.

La difficoltà sta nel fatto che il significato cosi come lo stile trova ormai la massima espressione nel testo originale che il traduttore debba dare il meglio di se a tradurlo in una lingua completamente diversa. Belloc (1931) sugerisce che il traduttore debba percepire il testo come unità strutturata. Nel caso il traduttore pensasse in questo modo, allora sarebbe molto più semplice a tradurre il testo. In più il traduttore non deve dimenticare le esigenze stilistiche e sintattiche del testo d'arrivo. In realta il traduttore dispone della liberta di modificare il testo durante la traduzione, conservando però le norme stilistiche e idiomatiche della lingua d'arrivo. La traduzione cosi sarebbe meglio accettata dal lettore nella lingua d'arrivo. I dati tratti dall'opera di Dreiser tradotta in albanese, sono classificate in due categorie: equivalenza e shift. 


\subsection{L'equivalenza formale}

Il soggetto, il predicato e i complementi vengono tradotti rispettivamente con soggetto, predicato e complementi nella lingua d'arrivo.

1. Stela was gone. - Stela kishte ikur.

2. A doctor had told him to drink hot water in the morning.- Një doktor $i$ kishte thënë të pinte në mëngjes ujë të ngrohtë.

3. Juxhini saw at once .- Juxhini e kuptoi menjëherë.

4. Where do you live? - Ku banoni?

5. Suzane Dale was young and beautiful. - Suzana Deili ishte e re dhe e bukur.

6. 'Oh, Angela,' he pleaded - 'Oh Anxhela,' iu lut ai.

7. We dont want anyone else, do we Peter? - Ne nuk duam njeri tjetër, apo jo Piter?

8. Could he really get Suzanne, if he went to Canada, to find her? - A do të mund ta merrte. Suzanën, po të shkonte në Kanada?

9. Sondra loves her Clyde so much. - Sondra e do fort Klajdin e saj.

10. She will come to me. - Ajo do të vijë vetë tek unë.

\subsection{L'equivalenza dinamica}

1. I was afraind you had stropped growing. - Kisha patur frikë se s'do të zgjateshe më.

2. This thing's getting the best of me. - Kjo po më ha kokën.

3. You are as cute as a doll. - Ju jeni per tu adhuruar.

4. That's a lie and it's out of the whole cloth. - Kjo është gënjeshtër, këmbë e krye gënjeshtër!

5. G'wan?- A ke gjë më për të gënjyer?

6. Have your bath and we'll talk about it afterwards. - Bëj një herë banjo dhe pastaj flasim.

7. You will not get a chance to do it again. - Një herë më gënjeve, por s'do të kesh mundësi ta bësh një herë tjetër.

8. Have you had supper?- she asked - A keni ngrënë darkë- e pyeti ajo.

9. And Arturo Scalchero-hullo, Skalger, you old frost!- Sa mirë që erdhët Arturo Skallxher, mik i dashur!

10. How are you, Mr.Witla? he inquired. - Si shkoni, mister Uitlla- e pyeti.

Analizzando le frasi si comprende che esse sono state tradotte attaverso l'equivalenza dinamica. Negli esempi 1-5 l'equivalente albanese per gli idiomi tipici della cultura e della lingua inglese non poteva essere tradotto attraverso l'equivalenza formale. L'equivalente dinamico che è stato utilizzato nella lingua d'arrivo ha il significato più vicino possibile a quello della parola o dell'espressione della lingua di partenza. Le frasi 6,7 e 8 presentano delle differenze a causa della struttura delle espressioni fisse in albanese e in inglese, ad esempio in inglese: ka një banjë (have a bath), ka darkë (have supper) apo merr një shans (get a chance) në shqip bën dush (do bath), ka ngrënë darkë (have eaten supper) apo ka një shans (have a chance). Le frasi 9 e 10 sono espressioni che vengono usate per salutare le persone. Secondo Newmark (1988), queste espressioni vengono usate per mantenere rapporti amichevoli tra le persone più che per scambiare informazione. Lui suggerisce che le sudette esppresioni devono essere tradotte con le loro equivalenti standard, le quali non è detto che debbano essere formali. Di coseguenza il traduttore ha tradotto la frase con l'equivalente dinamico, che ha nella lingua albanese la stessa funzione cha ha anche nell'inglese.

\section{Category Shifts}

Le frasi sono selezionate casualmente. In seguito sono analizzati shifts secondo le categorie a cui appartengono. Alla fine, sono tratte delle conclusioni in relazione alle zone di shifts utilizzate nella traduzione di Dreiser dall'inglese all' albanese. La realizzazione di structure shifts è annalizata in relazione ai componenti della frase: soggetto, complemento e predicato. L'attenzione in intra-system shift è focalizzata nella realizzazione attraverso la comparsa delle forme del singolare e del plurale in ambedue le lingue.

\subsection{Esempio.1}

If you love me, come and get me.

Nëqoftëse më dashuroni, ejani të më merrni. 


\subsubsection{Structure shifts}

Cambiamento nella struttura della frase

Frase subordinata - SVO (in inglese) - OV (in albanese)

If you love me, come and get me.

$$
\text { S V O V O }
$$

Nëqoftëse më dashuroni, ejani të më merrni.

$$
\text { O V V } 0
$$

\subsection{Esempio.2}

It never occurred to him that it was accidental, as indeed it was not, but only that it was a trick sharp, cunning, ill-timed for him, just the thing calculated to blast his career and tie for him down to the old regime when he wanted most to be free.

Për asnjë çast nuk i shkonte në mendje se kjo kish qenë gjë e rastit dhe në të vërtetë nuk gabohej, por se ishte një manover e ulët, e llogaritur, dinake, e përdorur me qëllim në çastin e duhur, për të prishur planet e së ardhmes dhe për ta mbajtur të lidhur në jetën e vjetër, pikërisht në çastin, kur donte të çlirohej më shumë se kurdoherë tjetër.

\subsubsection{Structure shifts}

L'aggiunta della congiunzione 'dhe' nella traduzione in albanese

It never occurred to him that it was accidental, as indeed it was not.

Për asnjë çast nuk i shkonte në mendje se kjo kish qenë gjë e rastit dhe në të vërtetë nuk gabohej.

Frase finite in originale $=$ Frase non-finite in albanese

(finite) just the thing calculated to blast his career

(non-finite) për të prishur planet e së ardhmes

\subsubsection{Unit shifts}

\section{- $\quad$ Espressione $=$ Parola o Parola $=$ Espressione}
it was not-gabohej
accidental- gjë e rastit
never- për asnjë cast when- pikërisht në castin
occur - $i$ shkonte në mendje
indeed- në të vërtetë
career- planet e së ardhmes
ill-timed - e përdorur me qëllim në çastin e duhur

\subsection{Esempio.3}

The introduction of this astounishing fact in connection with Angela was so unexpected, so morally diverting and peculiar that though Eugene denied it, half believed she was lying, he was harassed by the thought that she might be telling the truth.

Fjalët tronditëse që $i$ kishte thënë mënjanë Anxhela, qenë kaq të papritura dhe të rëndësishme, saqë Juxhini nuk deshi t'i besonte dhe, mëgjithëse qe gati i bindur se nuk ishin të vërteta, prapëseprapë e brente dyshimi se ajo mund të kish thënë të vërtetën.

\subsubsection{Structure shifts}

- $\quad$ La frase nominale in originale $=$ Frase subordinata relativa in albanese

The introduction of this astounishing fact in connection with Angela

Fjalët tronditëse që $i$ kishte thënë mënjanë Anxhela

- Frase complessa in originale $=$ Frase semplice in albanese 
Half believed she was lying.

Ajo mund të kish thënë të vërtetën.

- L'aggiunta della congiunzione 'dhe','megjithëse' e 'prapseprapë' nella traduzione in albanese

...denied it, half believed she was lying, he was harassed by the thought that she might be telling the truth. ..dhe, mëgjithëse qe gati i bindur se nuk ishin të vërteta, prapëseprapë e brente dyshimi se ajo mund të kish thënë të vërtetën.

\subsubsection{Unit shifts}

La traduzione di una parola con un' espressione

dinied- nuk deshi ta besonte

\subsubsection{Intra-sytem}

La traduzione di un nome al singolare con un nome al plurale

the introduction- fjalët

\subsection{Esempio.4}

It was so unfair, though, was all he could think, so unkind!

I dukej se sjellja e saj kish qenë shumë e padrejtë, tepër mizore.

\subsubsection{Structure shifts}

- Shift nella posizione della frase principale e di quella subordinata

$$
\text { It was so unfair, thou gh, was all he could think, so unkind! }
$$

\subsubsection{Unit shifts}

La traduzione di una parola con un' espressione o espressione con una parola

unkind - tepër mizore

all he could think- $i$ dukej

\subsection{Esempio. 5}

Beyond the reach of our intelligence as are the mysteries of the objects known by our senses, those presented in this universal matrix are, if we may say so, still further beyond the reach of our intelligence, for whereas, those of the one kind may be, and are, thought of by many as explicable on the hypothesis of creation, and by the rest on the hypothesis of the evolution, those of the other kind cannot by either be regarded as thus explicable.

Përtej caqeve që arrin mendja jonë, njohjes së sendeve që kapin shqisat tona, të fshehtat që kanë të bëjnë me rregullimin e gjithësisë janë të paarritshme për mendjen tonë, sepse, ndërsa të parat mund të konsiderohen-dhe, në të vërtetë, konsiderohen-të shpjegueshme me anë të hipotezës së krijimit të botës ose me hipotezën e evolucionit, të tjerat nuk e gjejnë një shpjegim të tillë.

\subsubsection{Structure shifts}

- Cambiamento nella posizione della proposizione principale, di quelle subordinate e nella tipologia delle proposizioni

- $\quad$ are the mysteries of the objects...(frase principale coordinata) 
- $\quad$ known by ourselves ...(frase implicite, modifica il nome 'objects')

- $\quad$ those presented in this universal matrix are still further beyond the reach of our intelligence...(frase principale coordinata)

- $\quad$ if we may say so...(frase condizionale dipendente dalla frase principale)

- $\quad$ for whereas, those of one kind may be...(frase causale dipendente dalla frase principale)

- $\quad$ and are thought of by many as explicable...(frase causale coordinata con la prima frase)

- $\quad$ those of the other kind cannot by either regarded as...(una frase causale dipendente dalla frase principale)

La frase nella traduzione albanese ha una struttura diversa:

- Përtej caqeve të njohjes së sendeve (non e' collegata ad altre parti)

- që arrin mendja jonë (frase subordinate relative)

- që kapin shqisat tona.... (frase subordinate relative)

- të fshehtat janë të paarritshme për mendjen tonë...(frase principale)

- që kanë të bëjnë me rregullimin e gjithësisë (frase subordinate relative)

- ndërsa të parat mund të konsiderohen me anë të hipotezës së krijimit të botës ose me anë të hipotezës së evolucionit (frase subordinate causale)

- dhe në të vërtetë konsiderohen... (frase coordinate)

- sepse, të tjerat nuk e gjejnë një shpjegim të tillë (subordinate causale)

La ripetizione delle frasi subordinate le quali si riferiscono allo stesso oggetto, l'uso frequente del pronome dimostrativo those: the objects known by our senses; those presented in this universal matrix; those of one kind, those of the other kind, così come la ripetizione della stessa locuzione avverbiale beyond the reach of our intelligence, pongono dei problemi nel ricostruire le stesse strutture dell'originale. Per questa ragione il traduttore ha scelto la traduzione sinonimica delle locuzioni avverbiali beyond the reach of our intelligence, sostituendole in albanese con la frase subordinata relativa 'përtej caqeve që arrin mendja jonë' che è l'equivalente semantico della frase principale 'janë të paarritshme për mendjen tonë', e hanno in qualche modo danneggiato la fluidità nella lingua albanese (mendja jonëmendjen tonë) comportando delle difficoltà nella comprensione del periodo nella sua interezza specie nella sua scomposizione semantica (è difficile capire quali sono 'të parat'e quali sono 'të tjerat').

La scelta della perifrasi per evitare l'uso frequente delle congiunzioni così come la scelta accurata delle congiunzioni indispensabili hanno attenuato il problema consentendo la conservazione del nucleo semantico - logico delle parti nella traduzione. Applicando la modulazione (are further beyond our reach-janë të pakapshme për mendjen tonë')e la trasposizione (the reach (n)- 'që arrin' (frase subordinate relative) prezented (v)- 'që kanë të bëjnë' (frase subordinate relative); explicable (adj)- 'shpjegim' (n)., ecc) il traduttore ha riportato le frasi senza alterare il loro significato semantico. Purtroppo, il contrasto tra by many e by the rest viene perso nella traduzione. Si puo dire altresì che l'uso nella lingua d'arrivo della congiunzione disgiuntiva 'ose' anziché della congiuzione copulativa 'dhe' ha alterato il significato dell'originale: thought of by many as explicable on the hypothesis of creation, and by the rest on the hypothesis of the evolution -'të shpjegueshme me anë të hipotezës së krijimit të botës ose me hipotezën e evolucionit'.

Inoltre la frase subordinata causale those of one kind may be... insieme alla frase incompleta con essa coordinata attraverso la congiunzione 'dhe', sono riportate in albanese con la frase causale 'sepse ndërsa të parat mund të konsiderohen dhe në të vërtetë, konsiderohen..', nella struttura della quale si nota la presenza di una frase incidentale 'në të vërtetë konsiderohet,' che poteva anche essere evitata perchè, nonostante il cambiamento dell'ordine delle frasi all' interno del periodo abbia messo ancor più in evidenza la veridicità dell'affermazione dichiarata, in qualche modo ha causato delle difficoltà nella comprensione e presentazione dell'informazione in conformità con la natura della lingua albanese. Diventiamo ancora più insistenti, su questo punto, dato che la sintassi della lingua albanese permette un ordine sintattico flessibile. La traduzione sinonimica del pronome dimostrativo those con il pronome indefinito 'të tjerat,' il pronome relativo 'që,' il numerale 'të parat,' è un chiaro esempio di traduzione funzionale o contestualizzata.

\subsubsection{Unit shifts}

\section{Espressione $=$ Parola o Parola $=$ Espressione}

those of the other kind- të tjerat creation- krijimit të botës those of the one kind- të parat those- të fshehtat 


\subsubsection{Class shifts}

$$
\begin{aligned}
& \text { Aggettivo = Sostantivo } \\
& \text { explicable- një shpjegim }
\end{aligned}
$$

\subsubsection{Intra-system shifts}

Nome al plurale= Nome al singolare

mysteries- njohja

II nostro suggerimento sarebbe quello di iniziare il periodo con la frase principale per essere poi completato con le frasi subordinate, cosa che semplificherebbe la lettura e la comprensione da parte del lettore. Vorremmo suggerire questa traduzione:

'Njohja e sendeve të perceptueshme prej shqisave tona, si dhe e të fshehtave që kanë të bëjnë me rregullimin e gjithësisë, janë të paarritshme për mendjen tonë, pasi, ndërsa, të parat mund - dhe shpjegohen nga pjesa më e madhe me anë të hipotezës së krijimit dhe nga disa të tjerë me hipotezën e evolucionit, të fshehtat e tjera nuk e kanë një shpjegim të tillë.'

\subsection{Esempio.6}

She could not have understood if she could have seen into Eugene's mind at this time how her home town, her father's single farm, the blue waters of the little lake near her door, the shadows of the tall trees on her lawn were somehow compounded for him not only with classic beauty itself, but with her own charm.

Ajo nuk do ta kish kuptuar në atë kohë, po të kish pasur mundësi të shihte brenda mendjes së Juxhinit, i cili mendonte se qyteti i saj i vogël i lindjes, ferma e veçuar e të jatit, ujët e kaltër të liqenit të vogël pranë shtëpisë, hijet e pemëve te lëndina, përmblidhnin në sytë e tij jo vetëm bukurinë e tyre dhe, në këtë mënyrë, bëhej dhe më tërheqëse.

\subsubsection{Structure shifts}

- La traduzione delle frasi semplici in inglese con frasi complesse in albanese

were somehow compounded for him not only with classic beauty itself, but with her own charm përmblidhnin në sytë e tij jo vetëm bukurinë e tyre dhe, në këtë mënyrë, bëhej dhe më tërheqëse

4.6.2 Unit shifts

Congiunzione $=$ Frase subordinata relativa

how - $i$ cili mendonte se

\subsubsection{Class shifts}

Congiunzione coordinativa avversativa $=$ Congiunzione coordinativa copulativa

but- dhe

\subsubsection{Intra-system shifts}

Nome al plurale= nome al singolare

the blue waters- ujët e kaltër

Analizzando in modo più dettagliato la costruzione e la traduzione di questo periodo complesso possiamo notare come la traduzione in albanese contiene delle spiegazioni che mancano nel originale. Da una parte il traduttore ha 
introdotto le parole 'i lindjes', 'i vogël'; le espressioni: 'në sytë e tij,' 'dhe në këtë mënyrë,' 'bëhej dhe më,' e la frase subordinata:'i cili mendonte se' per dare delle spiegazioni in più per il lettore e per aiutarlo a comprendere meglio la situazione. Dall'altra parte, sono escluse dalla traduzione gli aggettivi: tall e classic i quali non incidono sul significato nella sua interezza.

\section{Conclusioni}

Nella traduzione letteraria, il traduttore non deve dimenticare che i significanti stilistici nella lingua di partenza possonno essere completamente diversi da quelli nella lingua d'arrivo. Per questa ragione il traduttore letterario non solo deve essere attento di trasmettere correttamente la forma (il lessico, la grammatica e la fonologia) ma anche di rimanere fedele alle caratteristiche letterarie del testo. "Per quanto riguarda il testo nella sua interezza risulta impossibile di transferire tutto il contenuto del testo originale nel testo d'arrivo"(Yinhua, 2011). In questo contesto è un obbligo per il traduttore che il significato non cambi ma sia lo stesso in entrambe le lingue.

L'analisi delle frasi in inglese tratte dalle opere di Dreiser e dei loro equivalenti albanesi dimostra che l'equivalenza formale è usata raramente e solo per la traduzione di frasi semplici, mentre nella maggior parte dei casi viene utilizzata l'equivalenza dinamica. Eugene A. Nida e C. Taber (1982) sottolineano che spesso la forma del testo originale viene cambiata, ma finché i cambiamenti seguono la regola di back transformation nella lingua originale, della continuità contestuale e della trasformazione nella lingua d'arrivo, il messaggio viene considerato fedelmente riportato. In questo studio si sottolinea che l'equivalenza dinamica viene utilizzata in casi di idiomi, saluti, espressioni fisse, o frasi che richiedono l'uso di tecniche come la modulazione, la trasposizione, l'espansione ecc, mantenendo nel testo d'arrivo lo stesso effetto del testo originale.

D'altra parte, l'analisi della traduzione delle frasi dall'inglese in albanese dimostra che i structure shifts sono presenti in quasi tutti i casi. Tuttavia ci sono stati dei casi eccezionali, come gli esempi 1.3.5. Secondo i dati dell' analisi, si puo dire che structure shifts e unit shifts possono essere considerati come due shifts necessari nel processo di traduzione dall'inglese in albanese dei periodi di Dreiser frasi composite perché al traduttore non viene lasciata molta scelta ed ogni altro cambiamento porterà a cambiare il suo stile. Unit e class shifts possono essere considerate come libere scelte. In altre parole, la scelta di unit e class shifts dipende dal fatto se il traduttore ha scelto la traduzione libera (free) o diretta (letterale), covert o over, semantico o comunicativo.

In conclusione, sulla base dei dati di questo studio, possiamo dire che nella traduzione dei periodi complessi dall'inglese all'albanese, shifts e l'equivalenza risulta che vengono utilizzati in ugual modo. Nell'uso dell'equivalenza dinamica, la forma cambia mentre il significato rimane lo stesso, e nel caso dell'uso di shifts si avverte il tentativo del traduttore di realizzare una traduzione di qualità, tra queste due lingue. In questo contesto, la traduzione in albanese delle opere di Dreiser potrebbe considerarsi una buona traduzione, perché le opere sono riportate al lettore con un linguaggio fluente attraverso i mezzi linguistici che fornisce la lingua albanese.

\section{References}

Belloc, H. (1931). On Translation. Oxford: The Clarendon Press.

Catford, J. C. (1965). A Linguistic Theory of Translation. London: Oxford University Press.

Dreiser, Th. (1964). An American Tragedy, New York: Penguin Group Press.

Dreiser, Th. (1965). Gjeniu. (Mahmund. B, Trans.).Tiranë: Globus. (Original work published 1954)

Dreiser, Th. (1969). Një Tragjedi Amerikane. (Doko, B).Tiranë: Ombra GVG. (Original work published 1964).

Dreiser, Th. (1954). The Genius. United States of America: The World Publishing Company.

Hadaj, G. (1984). Vërtetësia artistike e origjinalit në përkthim nëpërmjet karakterizimit gjuhësor. Nëntori, nr.8. Tiranë.

Bulo, J. (1984). Ligjërimi i Personazhit në Prozën e Sotme Shqiptare. Studime Filologjike, nr.8. Tiranë.

Newmark, P. (1988). A Textbook of Translation. New York: Prentice Hall International.

Nida, E. A. (1964). Toward a science of translating: With special reference to principles and procedures involved in Bible translatin. Leiden: E.J. Brill.

Nida, E. A., \& Taber, C. R. (1982). The Theory and Practice of Translation. Leiden: E.J. Brill.

Quirk, R., Greenbaum, S., Leech, G., \& Svartvik, J. (1987). A comprehensive grammar of the English language (5th ed.). London and New York: Longman.

Webster's Revised Unabridged Dictionary. (1913). Edited by Noah Porter. G \& C. Merriam Co.

Yinhua, X. (2011). Equivalence in Translation: Features and Necessity. International Journal of Humanities and Social Science, 1(10). 